\title{
Lessons, open questions, and future prospects
}

\author{
M. Eracleous ${ }^{1, a}$, S. Gezari², D. Grupe ${ }^{1}$, S. Komossa ${ }^{3}$, and R. Saxton ${ }^{4}$ \\ ${ }^{1}$ Department of Astronomy \& Astrophysics, The Pennsylvania State University, 525 Davey Lab, \\ University Park, PA 16803, USA \\ 2 Hubble Fellow, Department of Physics and Astronomy, Johns Hopkins University, \\ 3400 North Charles Street, Baltimore, MD 21218, USA \\ ${ }^{3}$ Max-Planck-Institut für Radioastronomie, Auf dem Hügel 69, 53121 Bonn, Germany \\ ${ }^{4}$ XMM SOC, ESAC, Apartado 78, 28691 Villanueva de la Cañada, Madrid, Spain
}

\begin{abstract}
We summarize some of the highlights of this workshop, the first of its kind dedicated to observing and modeling the tidal disruption of stars by black holes in the centers of galaxies (or star clusters). We review the lessons learned from recent observations and from theory and identify outstanding questions and areas where more theoretical and observational work is needed. We also consider upcoming observing facilities that can be used to study tidal disruption events and speculate what these new facilities may contribute to the field.
\end{abstract}

\section{INTRODUCTION}

Current and upcoming surveys for transients are expected to find tidal disruption flares from galactic nuclei in large numbers (perhaps in the thousands[14] when the LSST becomes operational). We can then examine the distribution of events in different galaxy types and use tidal disruptions as tools for a variety of purposes.

- We can test the assumptions of stellar dynamical models that predict the event rates. In particular, we can constrain the supermassive black hole mass spectrum and the relation of the black hole mass to the host galaxy properties. We can also evaluate the importance of a number of effects that can enhance the rates compared to the simplest scenario of an isolated black hole in a stellar system (e.g., mass segregation, the presence of a binary black hole or any other structure that modifies the stellar orbits; see the contributions by Alexander, Liu, Chen, Karas, Amaro-Seoane, and Cuadra in these proceedings).

- We can carry out spectroscopic followup of many events and identify the type of object that was disrupted (main sequence stars vs evolved stars). Thus we can probe the stellar populations in the nuclei of galaxies (and even globular clusters) and use the findings to improve further the dynamical models that predict disruption rates. With refined models for the dynamics and emission properties of the post-disruption debris we can carry out case studies of the light curves to infer the internal structure of the object that was disrupted (see the contribution by Lodato, Guillochon, and Gezari in these proceedings).

- Through spectroscopic followup observations, we can also evaluate the effect of such flares in photoionizing the interstellar medium of the host

a e-mail: mce@astro.psu.edu galaxy (see [2,7] and the contribution by Komossa in these proceedings).

- We can look for the disruption of white dwarfs (WDs) through their emission-line signature (see contribution by Clausen in these proceedings). Such events provide a strong indication of the existence of intermediate-mass (IMBH, $M \sim 100-10^{5} \mathrm{M}_{\odot}$ ) black holes in galaxies or star clusters, since more massive black holes cannot disrupt WDs outside of their event horizon. Such events have also been hypothesized to lead to detonation of core of the star and they may resemble supernovae.

- When a space-based gravitational wave observatory becomes available, we can hope to detect gravitational waves from tidal disruption events, especially one involving an IMBH and a WD (this would constitute an extreme-mass ratio inspiral event; see the contributions by Amaro-Seoane and Laguna in these proceedings). Thus we can verify the scenario through an independent set of tests and obtain estimates of the masses of the two objects, as well as the distance. In the same spirit, we can use the short-lived accretion-powered systems resulting from these events to carry out tests of general relativity (see contributions by Stone and $\mathrm{Yu}$ in these proceedings).

- We can investigate how accretion disks form by studying the temporal and spectral evolution of tidal disruption events. Moreover, as we have seen in the past year, at least some tidal disruption events are accompanied by the formation of a jet (e.g., Swift J1644+57), which resemble in many ways those observed in quasars. Therefore, we have an opportunity to study the "birth" of a jet, which can teach us a great deal about the physics of jets in general.

- With a better understanding of the physics and rate of tidal disruptions we can consider the role they play in fueling nuclear activity, especially in lowluminosity active galaxies (AGNs). We can also 
apply the same lessons to evaluate the rates of star-accretion disk collisions in luminous AGNs and examine their effects on the structure and observational properties of the accretion disk.

But before we can achieve the above goals we must develop robust tests for identifying tidal disruption events observationally. This was one of the main themes of this conference, which was probably the first ever dedicated to the process of tidal disruption, the resulting observational signatures, and the rates of such events.

Significant theoretical progress has been made in recent years on the tidal disruption itself and predictions of the observational signature. Moreover, the first data from transient surveys (such as PanSTARRs and the PTF) are becoming available, which are bound to yield new insights into the relevant physical processes and refined estimates of the event rates. Thus the conference was very timely.

In this paper, we attempt to summarize some of the lessons learned from this conference and to identify important outstanding questions, both observational and theoretical. We discuss how current and upcoming transient surveys will contribute to the detection and characterization of tidal disruption events, the importance and challenges of followup spectroscopic observations, and pay particular attention to the issue of eliminating impostors. We also highlight open theoretical questions whose resolution will inform observational searches for and validation of tidal disruption events.

\section{THE STATE OF THE OBSERVATIONS}

About 25 events have been identified so far, the majority of them in the X-ray band (see the contribution by Komossa in these proceedings). The initial giant soft X-ray flares seen with ROSAT faded by factors up to 6000, more than a decade after their discovery. They have little, if any, detectable X-ray emission today. More recently, X-ray events have been detected with Chandra, XMM-Newton, and Swift (see the contributions by Saxton, Grupe, Esquej, Lin, and Levan in these proceedings).

We are now entering an era where systematic surveys for transients are starting to uncover substantial numbers of events (see contributions by Gezari and Cenko in these proceedings). We can, therefore, look forward to obtaining well-sampled light curves in multiple optical bands, which will allow detailed studies of the events and comparisons with theory. A good example is PS1$10 \mathrm{jh}$, a candidate tidal disruption event whose light curves seem to agree with simple models around the peak of the flare but not during the rise nor at late times. Optical spectroscopy of this event has shown fading emission lines, which are predicted by models but observed only rarely (see further discussion in $\$ 4$ ). On the negative side, the current transient surveys discover a wide variety of objects (including many supernovae and other explosive variables, for example); selecting those transients that are good candidates for tidal disruption events and deserve immediate followup is a significant challenge that requires a large investment of observational resources.
Events discovered in the optical and near-UV have been observed in the X-ray band and found to be quite faint. Fitting their spectral energy distributions (SEDs) with blackbody models yields considerably lower temperatures than those inferred for the events discovered in X-rays; the inferred temperatures cluster in the range $(2-5) \times 10^{4} \mathrm{~K}$. These flares not only have similar colors but also very similar light curves (they have power-law shapes with similar indices). These UV-selected flares are, however, quite distinct from the X-ray selected flares in their temperatures. This may indicate a distribution of flare temperatures (a spread of about an order of magnitude), which may be related to the conditions of the event. In view of the small number of events observed so far and the sparsely sampled SEDs, we cannot yet determine if the distribution of temperatures is bimodal or whether the UV and X-ray observations preferentially discover events in two different temperature regimes.

A particularly exciting observational development was the discovery with the BAT instrument on Swift of the hard X-ray flare Swift J1644+57 and related events (for a summary, see the contribution by Levan in these proceedings). These flares have been suggested to be tidal disruption events that have produced jets directed close to the line of sight, analogous to newly born blazars. This discovery has sparked a healthy debate on the nature of the engine powering these sources and the physical processes within the proposed jets (see further discussion in $§ 5$ ).

\section{WORRYING ABOUT IMPOSTORS}

Before an outburst from the nucleus of an apparently inactive galaxy can be regarded as a tidal disruption event, one must evaluate alternative possibilities. This exercise can be difficult depending on the sampling of the light curve and the availability of ancillary data (such as optical spectroscopy and multi-wavelength photometry). The most common and most serious worry is the possibility that the nucleus was actually already active, even if the activity was "mild." However, a supernova in the vicinity of the nucleus or a microlensing event may also masquerade as a tidal disruption event. We discuss below these possibilities in more detail.

A Weak AGN. - Tidal disruption events represent, in essence, the onset of nuclear activity in a previously inactive galaxy. Therefore there is an inherent difficulty in distinguishing them from other accretion events. What we wish to determine in this case is the source of fuel for the accretion process and the duration of the accretion event. Therefore, optical spectroscopy and high-energy (UV and X-ray) observations at late times, after the decline of the flare, can be used to look for an underlying conventional active nucleus (see the contributions by Komossa and Gezari in these proceedings and references therein). Such observations have to be rather sensitive, however, since weaklyactive nuclei (such as those found in LINERs, which can give rise to moderately large flares[10]), may be difficult to detect against the starlight from the host galaxy[12]. 
A Highly Variable AGN. - Good progress has been made on understanding the level and characteristics of variability in active galaxies (see contribution by Siemiginowska in these proceedings). Two types of active galaxies are though to be very likely impostors since they can display rapid, large-amplitude X-ray variability: Narrow-Line Seyfert 1 galaxies and BL Lac objects. The former can be identified from their optical spectra (as discussed by Grupe in these proceedings), while the latter can be identified from their X-ray spectra and radio properties (their optical spectra may be misleading [5]).

A Supernova or Microlensing Event. - A supernova in the vicinity of the nucleus of the host galaxy can pass off as a tidal disruption flare especially if the light curve is not well sampled. As emphasized by Lawrence in these proceedings, a microlensing event (i.e., the microlensing of a background quasar by a star in a foreground galaxy), caught on the decline, may also be mistaken as a tidal disruption event (see also [11]). These two possibilities can be assessed using the optical colors of the flare, the evolution of colors with time, and the properties of the emission lines, if any are detected. The color of a supernova is redder than that of a tidal disruption flare (after extinction corrections) and becomes even redder with time[14], while microlensing events are achromatic and have symmetric light curves, and do not produce transient emission-lines.

Vice-versa, there is also a danger that we may dismiss unusual tidal disruption events because they do not match our a priori expectations. Even though we usually aim to eliminate supernovae before we can consider a flare as a tidal disruption candidate, a strong encounter of a WD with an IMBH may lead to detonation and resemble a supernova observationally[13]. Another possibility is that the observed emission from a jet formed as a result of a tidal encounter (as in the case of Swift J1644+57; see contributions by Shcherbakov, Zauderer, and CastroTirado in these proceedings, and references therein). It is also possible that light emitted in the interaction of the post-disruption debris with ambient gas in the immediate vicinity of the nucleus contributes significantly to what we observe (see contribution by Komossa in these proceedings). In the same spirit, the emission-line spectrum produced by the photoionization of ambient gas will resemble that of an AGN, confounding our attempts to verify tidal disruption flares spectroscopically. Finally, we should keep in mind that theoretical predictions for the observational appearance of tidal disruption events are not yet robust (see the discussion in $\S 4$, below) and we should keep an open mind when studying unusual transients.

Dealing with the challenge of identifying a tidal disruption event requires significant observational resources. Well-sampled, multi-wavelength light curves are of great help since the colors of the transient and the evolution of the colors with time can be used to test the supernova and microlensing hypotheses. Moreover, catching the event early and observing the rise of the flare is also valuable as microlensing events (and perhaps other impostors not mentioned here) have symmetric light curves. Confirming that an event is a tidal disruption based on the shape of the light curve in different bands is probably more difficult than eliminating impostors for theoretical reasons, as discussed further in $\$ 4$.

Observations in high-energy bands (UV or X-ray) not only provide confirmation of the amplitude of the flare but they can reveal flares much more easily since the host galaxy is not as bright in those bands as it is in the optical. High-energy observations are also invaluable in defining the SED of the transient (the bulk of the power is emitted in these bands), which in turn allows us to study its energetics and constrain models for its nature (see the contributions by Komossa and Gezari in these proceedings).

Equally important is multi-epoch optical spectroscopy. Early time spectroscopy can take advantage of the brightness of the transient and help us determine if this is a candidate tidal flare that is worth monitoring further. Moreover, emission lines from the debris will be brightest and easiest to detect at early times. Such emission lines will tell us about its composition, hence the properties of the disrupted star, and provide additional tests for the nature of the flare (e.g., by identifying a supernova). For example, prompt spectroscopic follow-up of PS110jh detected fading, broad He II emission lines and no hydrogen, which revealed that the star disrupted was not an ordinary main-sequence star, but a helium-rich core of a star that had lost its hydrogen envelope. A similar conclusion was drawn for SDSS J074820.67+471214.3, which had very strong $\mathrm{He}$ II emission lines, and was interpreted by [15] as the tidal disruption of the Helium rich core of a star). Spectroscopy at intermediate times, when the flare is past its peak, can yield a redshift, hence a distance, that will enable us to study the energy budget of the event. Spectroscopy at late times, well after the flare subsides, is just as important for diagnosing if the nucleus of the host galaxy is active and determining the likelihood that the flare is a result of an instability in a preexisting accretion flow (see discussion in the contributions by Komossa and Gezari in these proceedings).

\section{MEANWHILE, ON THE THEORY FRONT...}

In the past five years, there have been significant advances in the theory of the evolution of the tidal disruption debris. Luminet, Lodato and Guillochon have presented sophisticated calculations of the disruption of the star and the subsequent fallback of the debris (see their contributions in these proceedings and references therein). In summary, the fallback rate is found to be proportional to $t^{-5 / 3}$ only at late times and only in a fraction of the cases studied. At early times, immediately after the peak, the fallback rate declines much more steeply than $t^{-5 / 3}$. The details of the behavior around the peak (rise time, peak rate, rate of decline past the peak) depend on the internal structure of the disrupted star. Of course the fallback rate does not directly translate into accretion rate or luminosity in any given band. The accretion disk, presumed to form out of the returning debris, is initially fed at a superEddington rate, while at late times it has a time-dependent temperature structure, dictated by the declining fallback rate. Moreover, there may also be UV emission from the 
outflowing gas (generated via internal shocks) during the super-Eddington phase (see contribution by Strubbe in these proceedings and references therein). As a result, the light curves in the optical and UV bands are considerably steeper than $t^{-5 / 3}$ after the peak of the flare and during the super-Eddington phase and considerably flatter than $t^{-5 / 3}$ after the super-Eddington phase [8,14].

In view of the above, it is puzzling that the observed light curves in the optical, UV, and X-ray bands are often observed to decline as $t^{-5 / 3}$ (see the reviews by Gezari and Komossa and other presentations of individual cases in these proceedings). Particularly intriguing is the $t^{-5 / 3}$ decline observed over the course of a decade in NGC 5905[6]. Similarly, the SEDs can be adequately described by a single-temperature blackbody model, when we should expect a multi-temperature black body model, representative of emission from a disk, or something more involved. Although the disk covers a small range of radii initially, it will quickly spread out to transport angular momentum with the result of spanning a wide range in temperatures (see the contribution by Shen in these proceedings). It is possible, of course, that single-temperature blackbody models are adequate only because the SEDs are not sampled well enough by current observations. On the other hand, these results may signal shortcomings in our understanding of the emission processes in these flares.

Indeed there is a gap between what current calculations predict, the debris fallback rate, and what observations can measure, the luminosity emerging from the accretion flow. We are in dire need of more sophisticated models for the production and propagation of photons in the accretion flow (including emission during the initial, superEddington phase). These should be based on a more detailed treatment of the formation of the accretion disk and its time dependent structure (see discussion by Shen in these proceedings). In effect, this problem is equivalent to computing the SED of a quasar, which has not yet been accomplished. The current observational results on the light curve and SED shapes lead one to suspect that there is an emission component that we do not fully appreciate: Perhaps we are observing the dissipation of the potential energy of the returning debris in shocks as matter is incorporated into the accretion flow; in such a case the emerging luminosity would track the debris return rate closely and, if the shocks are deeply buried, the resulting SED would resemble a single-temperature blackbody. Another possibility is that we are observing the photosphere of an outflow during the super-Eddington phase (see also [9]).

In the past five years there have also been calculations of the emission-line spectrum resulting from the illumination of the debris by the high-energy photons from the accretion flow (see contributions by Strubbe and Clausen in these proceedings). These calculations treat the photoionization problem very carefully but they are based on simple, analytical models for the evolution of the accretion rate and the geometry of the debris. In the case of the disruption of a solar-type star, the emissionline spectrum resembles that of a quasar, with broad, permitted emission lines from species with a wide range of ionization. The detailed predictions of the evolution of the emission-line spectrum with time are subject to the uncertainties on the SED and light curve of the ionizing light discussed above. However, the basic features of the emission-line spectrum (relative emission-line strengths, abrupt appearance and slow decline of the lines) are relatively robust predictions of these calculations. As such, the emission lines are a very useful tool for studying the composition of the debris. Nonetheless, emission lines are detected only rarely, which may be a result of their low contrast relative to the underlying continuum at late times.

\section{JETS FROM TIDAL DISRUPTION EVENTS}

The discovery of Swift J1644+57 and Swift J2058.4+0516 has underscored the possibility that jets are associated with tidal disruption events (see, for example, the contributions by Levan and Cenko in these proceedings and references therein). This development is not a surprise by any means since jets are an integral component of accretionpowered systems. This discovery has also prompted the development of models for the emission from such jets, as discussed by Piran, Metzger, and Shcherbakov in these proceedings. Jetted tidal disruption events afford us a rare opportunity to study the onset and eventually the disappearance of jets, which cannot be done in the case of AGNs with steady accretion rates. They also open the door to detecting tidal disruption events through their hard $\mathrm{X}$-ray and radio emission fro their jets under the right conditions. We may also hope to learn lessons about how the launching on jets depends on the properties of the black hole (mass and spin) by comparing these jets with the intermittent jets observed in black-hope X-ray binaries, which have been well studied for about a decade (see, for example, [3]).

\section{EPILOGUE}

In the next decade, transient surveys will produce a large number of tidal disruption candidates that will allow us to pursue the goals outline in $\S 1$. But to capitalize on these results, we will need to devote a great deal of observing time for photometric and spectroscopic followups. There is a real danger that the number of transients (of all types) will be too large to allow extensive spectroscopic followups. To cope with the anticipated volume of data we will have to make use of advanced statistical tools, such as machine learning algorithms, that will allow us to discriminate between different types of transient events early on in their evolution based on the photometric data delivered by the surveys. Similarly, we also need to carry out space-based followup observations in the UV and $\mathrm{X}$-ray bands; here too the available resources may not be adequate for the task. With the above considerations in mind, an X-ray instrument capable of monitoring large portions of the sky will be extremely useful in the study of tidal disruption candidates. Not only can it provide X-ray measurements to complement those at longer wavelengths, but it can also discover hard X-ray 
flares akin to Swift J1644+57, allowing us to explore the jet connection further. Searches for transients in the radio band (e.g., with LOFAR, see [4]) will also be quite useful since they can discover emission from jets formed during tidal disruption events.

Significant progress on the theory front is also needed before we can exploit the wealth of information encoded in the data. Given the theoretical uncertainties discussed in $\S 4$, we are now faced with the following difficult situation: we cannot be confident about our identification of a tidal disruption event until we have a good model for the light curves and SED, but we cannot validate our models without a secure identification and careful monitoring of at least a few events. In other words, we can use a Rosetta stone: a nearby and bright event that we can study in great detail.

\section{References}

[1] Cannizzo J. K., Lee H. M., \& Goodman J., 1990, Ap. J., 351, 38

[2] Eracleous, M., Livio, M., \& Binette, L. 1995, Ap. J., 445, L1
[3] Fender, R. \& Belloni, T. 2004, A. R.,A. EA., 42, 317

[4] Fender, R. 2012 in New Horizons in Time-Domain Astronomy, eds. E. Griffin, R. Hanisch, \& R. Seaman, IAU Symposium, 285, 11

[5] Halpern, J. P., Eracleous, M., \& Forster, K. 1997, A. J., 114, 1736

[6] Halpern, J. P., Gezari, S., \& Komossa, S. 2004, Ap. J., 604, 572

[7] Komossa, S. et al. 2008, Ap. J., 678, L13

[8] Lodato, G. \& Rossi, E. M. 2011, M.N.A.R.A.S., 410, 359

[9] Loeb, A. \& Ulmer, A. 1997, Ap. J., 489, 573

[10] Maoz, D., Nagar, N. M., Falcke, H., \& Wilson, A. S. 2005, Ap. J., 625, 699

[11] Meusinger, H. et al. 2010, AEFA, 512, A1

[12] Moran, E. C., Filippenko, A. V., \& Chornock, R. 2002, Ap. J., 579, L71

[13] Rosswog S., Ramirez-Ruiz E., \& Hix W. R., 2008, Ap. J., 679, 1385

[14] Strubbe, L. E. \& Quataert, E. 2009, M. N.A. R. A. S., 440, 2070

[15] Wang, T.-G., Zhou, H.-Y., Wang, L.-F., Lu, H.-L., \& $\mathrm{Xu}$, D. 2011, Ap.J., 740, 85 